TgCatJpOk3

$>$ ROP5 (consensus sequence)

ATGGCGACGAAGCTCGCTAGACTAGCCACGTGGCTTGTCTTGGTAGGTT GCCTGTTGTGGCGGGCGGGGGCAGTTCAGCTCTCTCCGCCAAACTCCA GGACGAATGATCTGGCTTCGGGAACCCCGCATGTGGCTCGTGGGGACA CTGAGGCGCAGTCAGGAACTGGAGACGATTCAGATTTCCCGCAGGGCG TGGTCGAAGAGGTGGCAGATATGAGCGGCGGCAGAGTTCCCCGAGTGC CAGCATCGTCTACCACCACATCTGCGTCCGAGGGGATTTTCAGAAGATT AGTTCGCAGACTTCGTCGGGGAAGAGGAACCGCAGATGGCGCAGGAGT TGCTGACGAAACCCATCAGGGGCCGCGCCCGCCACTTCGGAAGAGACT TGCTCAGCACTTCCGTAGGCTGAGGGGCTTCTTCGGACGCCTTACGCC GAGGTGGCTCTCCGGTCTCGGCCGCCGGGCGCAAAGATGGTGGAGAG GGAGACAGAGACCGCTGCTGGACCCTTCGTTTCATGGGTTGGAAGCTG GAGATTCGTTCATGCGCGACCTGCTGAAACGTGAAGAAGAGCTGATTG GATACTGTCGCGAAGAAGCGTTGGAAGAACCTGCAGCGATGGTTGAGG CTGTCATGGCAACTGTATGGCCGCAAAATGCTGAAACAACCGTGGATTC ACTTTTGAGTCAGGGAGAGCGGAAGTTGAAATTGGTGGAGCCTCTTCG AGTCGGTGACCGATCTGTCGTATTTTTAGTAAGGGATGTAGAGCGCCTG GAGGATTTCGCTCTGAAGGTCTTCACTATGGGTGCCGAGAATTCCCGAT CAGAGCTGGAGCGGTTGCATGAAGCGACTTTTGCGGCAGCGAGGTTGC TTGGGGAGAGTCCAGAGGAGGCACGGGACAGACGCAGGCTTTTACTTC CCTCCGATGCTGTGGCAGTTCAGTCTCAGCCCCCTTTCGCTCAGCTGAG TCCAGGACAGAGCGACTATGCAGTCGCGAACTATTTCTTTCTCATGCCC GCTGCGTCGGTGGATCTTGAATTGCTCTTTAGGACATTGGACTTCGTGT ATGTATTCAGGGGGGAAGAAGGTATTTTAGCGCGTCACTTACTAACGGC ACAGCTGATCCGTCTGGCAGCCAACCTGCAGAGCAAAGGACTTGTGCA TGGACGCTTCACACCTGAAAACCTTTTCATTATGCCCGATGGCCGCCTG ATGATGGGGGATGTATCCACGTTGAGGAAGGTCGGAACCCGAGGACCG GCATCAAGCGTCCCGGTTACCTATGCGCCTCGGGAGTTCTTGAATGCAA ACACGGCAACATTTACACACGCGCTCAATGCGTGGCAACTGGGTCTTAG CATATACCGGGTTTGGTGCCTAGTCTTGCCTTTCGGACTCGTGACACCT GGGATCAAACGGACATGGAAAAGGCCAAGTCTACGAGTTCCAGGGACT GACAGTCTGCTATTCGACTCATGTATACCTGTGCCTGACTTCGTGCAGA CACTTACTAGACGGTTCCTCAACTTCGATAGGCGGCGACGCCTGCTTCC CTTGGAGGCCATGGAGACGCCAGAGTTCCTCCAGCTCCAAAACGAAATA 
TCGAGCAGCCTATCAACAGGACAACCTACTGCTGCGCCCTCAGTCGCTT GA

$>$ ROP16

ATGAAAGTGACCACGAAAGgGCTTGCTTTTGCTCTTGCACTGTTGTTTT GTACACGCTGCGCAACTGCACGATACATGTCGTTTGAGGAAGCGCAAAA AGCAAGTGAAGCAGCGAAGCGCCAGATTGCCACACTCCCCTCTCCAGA TTCTACTCTTTCGAATCCAGGTAGCAAGCATAGAAACCGGGGAGGGTCT CCTGCGGCAGGGCAACCTTCTCAATCCACACTACAACCTGAACAAGCGG CGGCTGAAGTAGGTCTCGGTGCTGGTGGCTCGACTCAGGGGCAGGGAC GCACCGGTGGCAGCGCGGGTGCTAGAGAGGAGCGGAGGAGTCCTTCC CCCCAATCTGCTTATCCGGCGACTAGCTCAGCCTCGCTAAGGGGCTACC AAACCCAGCTTTCACCCTCGCATCTTCCACCACGCAGCAGCGGACCGG GAGGATGGTTTCCAACAGAGTCAATATTTACGCCATGGAGTTCTCCGCC GCAACCATTGACACAACGAAAGCCATCTCTATCTGGGGTGGTCGTTACC GAATTTCAAGAGCCACAAGAACAGTATGGCGCAGCGAGCAGTCTTGCG TCCTCGCCAAAGCGATACGTCAGTGGCGCAAGCTCGAGTGCATTGTCAG GAAAGGCGGTGCCAACGCCTGCGTCGCTTGGTCAAGAAAATCCTCTTTT CCCTGTTCAGAGCGCTACATTGGATTCAGGAATACAGTCTCCGGCACAA GAGCGTCGGGGATCCCCTCAAAGACAGATTGCGATGTCGACCGAAAAT CCAGCGGATAGCGGCGCCTCGCAGCTTGCCTCCAGTGTTTCTAGTTATG TAGCAGTACAAACTCCTCATGTGAAACGTTCAGAACGCATCCGGCGCGT TCGACTTTCAGAAGAGGGTCTGGAAGAAGTTCAGCAGCTGAAAGCAGC TGCCGCACAGCTTCTCGTAGCGGTTCCGGACTATGAGGCAATGCGGGCT GTTCTGCAAGAGGCGGTCCTCTCAGAACAGAGGGTTGCTACCCGTAAG CGGAAGAGAAAGCAACCTCCAGGAGCGGTGGAGTCAGCTGTTGACGAA GTGTTCCCTCCAAATGAGCGTGTCATGATGATAAATGCCAACGGAGTGC CGATCGCTCTATACAATCGTGGGCACCTCGGCAGTGGACATTTCGGGGC TGTCATCAAGGCCAGCTTAGACGATGGGACGTTGTATGCAGCGAAGGTG CCGTACAGCCAGATCGTCCCGAATGCTGATGCCACGTCAGCAGAACTGG AGgCGGAAATTTCCTCAGCTAGgGCGgAGTTGgTAAAGACAATTCGACA GGAGTTGGATGTTCGGGATAAGCTGGTGGCTAAAGGGCTCACACTTACA GAGACTGCGGAGCAATACGGTCTACCATTGTGCCAAATGACTTTAACGC TTCCTGAGAACAAAGCAACCGTGGTACGTCGAGGTTCTCGACTCGTTGT CGTGTCTAAAGAAGTCATGCTGCTGCCATTAATTGATGGCTCCCCATCG AACAGTCTAGTCCAGTCGCAACCACCATTTCTCTTCCAGCGAGCTGTGG 
CAAGGGAAGCAATTATTGCATTGGCCAAGCTTCACGAACTTGGATTCGC GCATGGAGATGTTAAATTGAACAACATGATGATCGATGTCCACGGCTTT GGGCATATGCTTGACATGGGCAGTGTGCGGCCTGTTGACAGCTGTGTAA GCGAGGAAGATAAATATTACCTGCGTTTGTGGGCTCCTGAACTTGCGAA ATCACAGCACACGTCGCAGCAGACATGTCTGAAGCGTGGCGCTCTCGAT GTGTGGGCCTTAGGGTTGGCAATCTTCGAGTTCGTCTGCTTCAACCGAC TTCCTTACAGCCTTTCGAATCTGCCGAGTTCACTCTGGTCGAGAGTTGA ACACCTTTCGCGCCTTCGCCTCTCAGATTTCTCTGCCAAGGATTGTAAC GAATCTGATCCAGCAGTGATGGGAATTGTTGCTCAATTTCTAАATCCAАA TCCTGAAGAGCGCCCTGAACTCCCGAAATTCGTCAGCAGTTACACCTTC TTTCGGCAAGCCCCTGGAGTTACTTCTCATCTCACTAGGATTCCAACTAC CGAACTTTCTTCACATCGGATGTAG

\section{$>$ ROP18}

ATTCGGGGCAAGAAAGAAGGAAGAAAATAAAGACGAGCGAGCACGCGGAC TCCTCGGTTCCCTCGTTCGAGCTAGCGACTGCGGGAGAACCACATCTTTGGC CCGAGATTCGAAACGCGGAAGTAACTCGAGTCGATGCCTCGCTAGATGGCGA CCAGAAAAGAAAACGAGTGAACTCTTGAAAGCAGCACCCTGAGGAGACAG CCAGGAAAGAAGGGACAGGAAGAAACTCGAGAGAAGCGGCAGGAGACTGT CACAGCTCGTCGACCACACAGCTAAACTGCCCGCCCTCTTTCATTAAGTCCC GTGTTTGAACGACGTCCGGGAGTGCCTTTTTCTTTTCGTTTCCCGACGTAGCA GCGAACCTAGCTGAATATTCCAGTTTCGCTTTGAGTTCATAACTCCTTCGTTC TCTCTCTTCCGCCCGTCAGTTCCCTTCCCTGGTGTCTTCGTGACATTTTATTAA GTTCTATCGCGCCACTCGGACTTTGTTCCTGGCACCCTTTTGTAGCTAGGACT CCTTGACGAGTCAGTCAGATTGACGAGGTCGGCTAGCTAGGACTCCTTGACG CGTCAGTCAGATTGACGAGGCCGGCTAGCTAGGACTCCTTGACGCGTCAGTC AGATTGACGAGGCCGGCTAGCCACGCTATGCACCTCTTGCATACAATTGTTGT AGACAGCATGGTGGTGTGCGAGACACTGCAGTCAAATGCCTCACCCAACGC CGCGTCTCATTCTTCCAAAAACCTGTCCGTGTCTTCGACAGATTGATACAGCC GTTGACAAAGCAATACCATATTTTACAGTTTTGTACTCACCCCAGTCCAGTTT GTGTGAAAGTTGTGATGTTTTCGGTACAGCGGCCACCTCTTACGCGTACC GTCGTCCGAATGGGTTTAGCGACTCTTCTCCCGAAGACAGCCTGTCTTG CGGTGTTAAATGTAGCGCTTGTCTTCCTGCTCTTCCAAGTCCAGGATGG GACCGGAATCACACTTGATCCTTCAAAACTCGACTCCAAACCGACAAGT TTGGATTCGCAACAGCACGTTGCTGACAAGCGGTGGCCTGCTACAGTT GGCCACTACAAATATTTAGCAGGAGCGACAGAAAGCACTCGAGACGTTT 
CATTGCTGGAGGAAAGGGCTCAACACCGGGTAAATGCGCAAGAAACAA ACCAACGGCGCACGATTTTTCAGAGGCTTCTGAATCTCTTGAGACGGAG AGAAAGAGATGGTGAAGTCTCGGGTTCCGCAGCTGATAGCTCCTCGAG ACCCCGTCTGTCCGTACGACAGAGGCTTGCTCAACTTTGGCGTAAAGCG AAATCGTTCTTCACACGCGGAATCCCGAGGTACTTTTCTCAAGGGCGTA ACCGACTGCGAAGTTTGCGGGCACAAAGACGGCGATCTGAATTGTTTTT TGAGAAGGCGGATTCTGGATGCGTCATCGGCAAACGCATCCTGGCGCA CATGCAAGAACAAATCGGGCAGCCTCAAGCGCTAGGAAATAGTGAACGA CTGGATAGAATTCTGACTGTCGCTGCCTGGCCTCCGGACGTTCCAGAAA GATTTGTTTCTGTGACTACCGGTGAAACCCGGACGCTGGTGAGAGGTG CACCCCTTGGCTCTGGTGGATTCGCCACTGTATATGAGGCTACAGACGT GGAGACGAATGAAGAGTTGGCTGTTAAGGTTTTCATGTCAGAAAAGGA GCCCACCGATGAGACTATGCGTGACTTGCAGAGGGAGTCGTTCTGCTAC AGGAACTTTAGTCTAGCCAAGACGGCGAAGGATGCCCAGGAACGCTGT AGATTCATGGTTCCTAGTGATGTTGTGATGTTAGAGGGACAGCCAGCAT CCACAGAGGTCGTGATTGGTTTGACGACTCGGTGGGTACCAAACTATTT TCTTCTCATGATGCGGGCAGAAACGGACATGAGCAAAGTCATTTCATGG GTATTTGGAGATGCGTCTGTCAATAACAGTGAATTAGGCCTGGTCGTTC GAATGTACCTATCCAGTCAGGCAATCAGACTAGTGGCCAATGTTCAAGC TCAGGGAATTGTGCATACGGATATCAAACCGGCGAATTTCCTCCTCTTG AAAGACGGTCGCCTGTTTCTCGGCGACTTCGGAACGTATAGAATCAATA ATTCGGTTGGACCCGCGATAGGTACTCCCGGTTACGAGCCTCCGGAGCG ACCGTTTCAGACTACAGGCATCACCTATACATTCACCACTGACGCGTGG CAACTCGGTATAACTTTGTACTGCATCTGGTGCAAGGAACGTCCAACTC CGGCCGACGGCATCTGGGACTACTTACACTTCGCAGATTGTCCTTCCAC GCCTGAGCTGGTTCAAGACCTCATCCGAAACCTCTTGAATCGAGAGCCT CAGAAACGGATGCTCCCGCTACAAGCCTTGGAGACCGCAGCGTTTAAC GAGATGGATTCAGTAGTAAAACGCGCCGCGCAAAACTTCGAACAGCAG GAACATCTCCACACAGAATAA 
TgCatJpOk4

$>$ ROP5 (consensus sequence)

ATGGCGACGAAGCTCGCTAGACTAGCCACGTGGCTTGTCTTGGTAGGTT GCCTGTTGTGGCGGGCGGGGGCAGTTCAGCTCTCTCCGCCAAACTCCA GGACGAATGATCTGGCTTCGGGAACCCCGCATGTGGCTCGTGGGGACA CTGAGGCGCAGTCAGGAACTGGAGACGATTCAGATTTTCCGCAGGCCG TGGCCGAAGAGGTGGCAGATATGAGCGGCGGCAGAGTTCCCCGAGTGC CAGCATCGTCTACCACCACATCTGCGTCCGAGGGGATTTTCAGAAGATT AGTTCGCAGACTTCGTCGGGGAAGAGGAACCGCAGATGGCGCAGGAGT TGCTGACGAAACCCATCAGGAGCCGCGCCCGCCACTTCGGAAGAGACT TGCTCAGCACTTCCGTAGGCTGAGGGGCTTCTTCGGACGCCTTACGCC GAGGTGGCTCTCCGGTCTCGGCCGCCGGGCGCAAAGATGGTGGAGAG GGAGACAGAGACCGCTGCTGGACCCTTCGTTTCATGGGTTGGAAGCTG GAGATTCGTTCATGCGCGACCTGCTGAAACGTGAAGAAGAGCTGATTG GATACTGTCGCGAAGAAGCGTTGAAAGAACCTGCAGCGATGGTTGAGG CTGTCACGGCAACTGTATGGCCGCAAAATGCTGAAACAACCGTGGATTC GCTTTTGAGTCAGGGAGAGCGGAAGTTGAAATTGGTGGAGCCTCTTCG AGTCGGTGACCGATCTGTCGTATTTTTAGTAAGGGATGTAGAGCGCCTG GAGGATTTCGCTCTGAAGGTCTTCACTATGGGTGCCGAGAATTCCCGAT CAGAGCTGGAGCGGTTGCATGAAGCGACTTTTGCGGCAGCGAGGTTGC TTGGGGAGAGTCCAGAGGAGGCACGGGACAGACGCAGGCTTTTACTTC CCTCCGATGCTGTGGCAGTTCAGTCTCAGCCCCCTTTCGCTCAGCTGAG TCCAGGACAGAGCGACTATGCAGTCGCGAACTACTTGCTTCTCATGCCC GCTGCGTCGGTGGATCTTGAATTGCTCTTTAGCACATTGGACTTCGTGT ATGTATTCAGGGGGGCAGAAGATTTTTTAGCGCTTCACATACTAACGGC ACAGCTGATCCGTCTGGCAGCCAACCTGCAGAGCAAAGGACTTGTGCA TGGACATTTCACACCGGATAACCTTTTCATTATGCCCGATGGCCGCCTGA TGCTGGGGGATGTATCCGTGTTGAGGAAGGTCGGAACCCGAGGACCGG CATCAAGCGTCCCGGTTACCTATGCGCCTCGCGAGTTCTTGAATGCAAG CACGGCAACATTTACACACGCGCTGGATGCGTGGCAACTGGGTCTTAGC ATATACCGGGTTTGGTGCCTATTCTTGCCTTTCGGACTCGTGACACCTG GGATCAAAGGGTCATGGAAAAGACCAAGTCTACGAGTTCCAGGGACTG ACAGTCTGGCATTCGGCTCATGTACACCTCTGCCTGACTTCGTGCAGAC ACTTATTGGACGGTTCCTCAACTTCGATAGGCGTCGACGCCTGCTTCCC TTGGAGGCCATGGAGACGCCAGAGTTCCTCCAGCTCCAAAACGAAATAT CGAGCAGCCTATCAACAGGACAACCCATTGCTGCGCCCTCAGTCGCTTG 
$\mathbf{A}$

$>$ ROP16

ATGAAAGTGACCACGAAAGGGCTTGCTTTTGCTCTTGCACTGTTGTTTT GTACACGCTGCGCAACTGCACGATACATGTCGTTTGAGGAAGCGCAAAA AGCAAGTGAAGCAGCGAAGCGCCAGATTGCCACACTCCCCTCTCCAGA TTCTACTCTTTCGAATCCAGGTAGCAAGCATAGAAACCGGGGAGGGTCT CCTGCGGCAGGGCAACCTTCTCAATCCACACTACAACCTGAACAAGCGG CGGCTGAAGTAGGTCTCGGTGCTGGTGGCTCGACTCAGGGGCAGGGAC GCACCGGTGGCAGCGCGGGTGCTAGAGAGGAGCGGAGGAGTCCTTCC CCCCAATCTGCTTATCCGGCGACTAGCTCAGCCTCGCTAAGGGGCTACC AAACCCAGCTTTCACCCTCGCATCTTCCACCACGCAGCAGCGGACCGG GAGGATGGTTTCCAACAGAGTCAATATTTACGCCATGGAGTTCTCCGCC GCAACCATTGACACAACGAAAGCCATCTCTATCTGGGGTGGTCGTTACC GAATTTCAAGAGCCACAAGAACAGTATGGCGCAGCGAGCAGTCTTGCG TCCTCGCCAAAGCGATACGTCAGTGGCGCAAGCTCGAGTGCATTGTCAG GAAAGGCGGTGCCAACGCCTGCGTCGCTTGGTCAAGAAAATCCTCTTTT CCCTGTTCAGAGCGCTACATTGGATTCAGGAATACAGTCTCCGGCACAA GAGCGTCGGGGATCCCCTCAAAGACAGATTGCGATGTCGACCGAAAAT CCAGCGGATAGCGGCGCCTCGCAGCTTGCCTCCAGTGTTTCTAGTTATG TAGCAGTACAAACTCCTCATGTGAAACGTTCAGAACGCATCCGGCGCGT TCGACTTTCAGAAGAGGGTCTGGAAGAAGTTCAGCAGCTGAAAGCAGC TGCCGCACAGCTTCTCGTAGCGGTTCCGGACTATGAGGCAATGCGGGCT GTTCTGCAAGAGGCGGTCCTCTCAGAACAGAGGGTTGCTACCCGTAAG CGGAAGAGAAAGCAACCTCCAGGAGCGGTGGAGTCAGCTGTTGACGAA GTGTTCCCTCCAAATGAGCGTGTCATGATGATAAATGCCAACGGAGTGC CGATCGCTCTATACAATCGTGGGCACCTCGGCAGTGGACATTTCGGGGC TGTCATCAAGGCCAGCTTAGACGATGGGACGTTGTATGCAGCGAAGGTG CCGTACAGCCAGATCGTCCCGAATGCTGATGCCACGTCAGCAGAACTGG AGgCGGAAATTTCCTCAGCTAGGGCGGAGTTGGTAAAGACAATTCGACA GGAGTTGGATGTTCGGGATAAGCTGGTGGCTAAAGGGCTCACACTTACA GAGACTGCGGAGCAATACGGTCTACCATTGTGCCAAATGACTTTAACGC TTCCTGAGAACAAAGCAACCGTGGTACGTCGAGGTTCTCGACTCGTTGT CGTGTCTAAAGAAGTCATGCTGCTGCCATTAATTGATGGCTCCGCATTG AACAGTCTAGTCCAGTCGCAACCACCATTTCTCTTCCAGCGAGCTGTGG CAAGGGAAGCAATTATTGCATTGGCCAAGCTTCACGAACTTGGATTCGC 
GCATGGAGATGTTAAATTGAACAACATGATGATCGATGTCCACGGCTTT GGGCATATGCTTGACATGGGCAGTGTGCGGCGTGTTGACAGCTGTGTAA GCGAGGAAGATAAATATTACCTGCGTTTGTGGGCTCCTGAACTTGCGAA ATCACAGCACACGTCGCAGCAGACATGTCTGAAGCGTGGCGCTCTCGAT GTGTGGGCCTTAGGGTTGGCAATCTTCGAGTTCGTCTGCTTCAACCGAC TTCCTTACAGCCTTTCGAATCTGCCGAGTTCACTCTGGTCGAGAGTTGA ACACCTTTCGCGCCTTCGCCTCTCAGATTTCTCTGCCAAGGATTGTAAC GAATCTGATCCAGCAGTGATGGGAATTGTTGCTCAATTTCTAAATCCAAА TCCTGAAGAGCGCCCTGAACTCCCGAAATTCGTCAGCAGTTACACCTTC TTTCGGCAAGCCCCTGGAGTTACTTCTCATCTCACTAGGATTCCAACTAC CGAACTTTCTTCACATCGGATGTAG

$>$ ROP18

ATTCGGGGCAAGAAAGAAGGAAGAAAATAAAGACGAGCGAGCACGCGGAC TCCTCGGTTCCCTCGTTCGAGCTAGCGACTGCGGGAGAACCACATCTTTGGC CCGAGATTCGAAACGCGGAAGTAACTCGAGTCGATGCCTCGCTAGATGGCGA CCAGAAAAGAAAACGAGTGAACTCTTGAAAGCAGCACCCTGAGGAGACAG CCAGGAAAGAAGGGACAGGAAGAAACTCGAGAGAAGCGGCAGGAGACTGT CACAGCTCGTCGACCACACAGCTAAACTGCCCGCCCTCTTTCATTAAGTCCC GTGTTTGAACGACGTCCGGGAGTGCCTTTTTCTTTTCGTTTCCCGACGTAGCA GCGAACCTAGCTGAATATTCCAGTTTCGCTTTGAGTTCATAACTCCTTCGTTC TCTCTCTTCCGCCCGTCAGTTCCCTTCCCTGGTGTCTTCGTGACATTTTATTAA GTTCTATCGCGCCACTCGGACTTTGTTCCTGGCACCCTTTTGTAGCTAGGACT CCTTGACGAGTCAGTCAGATTGACGAGGTCGGCTAGCTAGGACTCCTTGACG CGTCAGTCAGATTGACGAGGCCGGCTAGCTAGGACTCCTTGACGCGTCAGTC AGATTGACGAGGCCGGCTAGCCACGCTATGCACCTCTTGCATACAATTGTTGT AGACAGCATGGTGGTGTGCGAGACACTGCAGTCAAATGCCTCACCCAACGC CGCGTCTCATTCTTCCAAAAACCTGTCCGTGTCTTCGACAGATTGATACAGCC GTTGACAAAGCAATACCATATTTTACAGTTTTGTACTCACCCCAGTCCAGTTT GTGTGAAAGTTGTGATGTTTTCGGTACAGCGGCCACCTCTTACGCGTACC GTCGTCCGAATGGGTTTAGCGACTCTTCTCCCGAAGACAGCCTGTCTTG CGGTGTTAAATGTAGCGCTTGTCTTCCTGCTCTTCCAAGTCCAGGATGG GACCGGAATCACACTTGATCCTTCAAAACTCGACTCCAAACCGACAAGT TTGGATTCGCAACAGCACGTTGCTGACAAGCGGTGGCCTGCTACAGTT GGCCACTACAAATATTTAGCAGGAGCGACAGAAAGCACTCGAGACGTTT CATTGCTGGAGGAAAGGGCTCAACACCGGGTAAATGCGCAAGAAACAA 
ACCAACGGCGCACGATTTTTCAGAGGCTTCTGAATCTCTTGAGACGGAG AGAAAGAGATGGTGAAGTCTCGGgTTCCGCAGCTGATAGCTCCTCGAG ACCCCGTCTGTCCGTACGACAGAGGCTTGCTCAACTTTGGCGTAAAGCG AAATCGTTCTTCACACGCGGAATCCCGAGGTACTTTTCTCAAGGGCGTA ACCGACTGCGAAGTTTGCGGGCACAAAGACGGCGATCTGAATTGTTTTT TGAGAAGGCGGATTCTGGATGCGTCATCGGCAAACGCATCCTGGCGCA CATGCAAGAACAAATCGGGCAGCCTCAAGCGCTAGGAAATAGTGAACGA CTGGATAGAATTCTGACTGTCGCTGCCTGGCCTCCGGACGTTCCAGAAA GATTTGTTTCTGTGACTACCGGTGAAACCCGGACGCTGGTGAGAGGTG CACCCCTTGGCTCTGGTGGATTCGCCACTGTATATGAGGCTACAGACGT GGAGACGAATGAAGAGTTGGCTGTTAAGGTTTTCATGTCAGAAAAGGA GCCCACCGATGAGACTATGCGTGACTTGCAGAGGGAGTCGTTCTGCTAC AGgAACTTTAGTCTAGCCAAGACGGCGAAGGATGCCCAGGAACGCTGT AGATTCATGGTTCCTAGTGATGTTGTGATGTTAGAGgGACAGCCAGCAT CCACAGAGGTCGTGATTGGTTTGACGACTCGGTGGGTACCAAACTATTT TCTTCTCATGATGCGGGCAGAAACGGACATGAGCAAAGTCATTTCATGG GTATTTGGAGATGCGTCTGTCAATAACAGTGAATTAGGCCTGGTCGTTC GAATGTACCTATCCAGTCAGGCAATCAGACTAGTGGCCAATGTTCAAGC TCAGGGAATTGTGCATACGGATATCAAACCGGCGAATTTCCTCCTCTTG AAAGACGGTCGCCTGTTTCTCGGCGACTTCGGAACGTATAGAATCAATA ATTCGGTTGGACCCGCGATAGGTACTCCCGGTTACGAGCCTCCGGAGCG ACCGTTTCAGACTACAGGCATCACCTATACATTCACCACTGACGCGTGG CAACTCGGTATAACTTTGTACTGCATCTGGTGCAAGGAACGTCCAACTC CGGCCGACGGCATCTGGGACTACTTACACTTCGCAGATTGTCCTTCCAC GCCTGAGCTGGTTCAAGACCTCATCCGAAACCTCTTGAATCGAGAGCCT CAGAAACGGATGCTCCCGCTACAAGCCTTGGAGACCGCAGCGTTTAAC GAGATGGATTCAGTAGTAAAACGCGCCGCGCAAAACTTCGAACAGCAG GAACATCTCCACACAGAATAA 